\title{
DETECCIÓN Y ANÁLISIS DE MOVIMIENTOS DEL TERRENO MEDIANTE TÉCNICAS D-INSAR EN LA ISLA DECEPCIÓN (ANTÁRTIDA)
}

\section{DETECTION AND ANALYSIS OF GROUND MOTION USING DINSAR TECHNIQUES ON DECEPTION ISLAND (ANTARCTICA)}

\author{
Rogelio de la Vega-Panizo a, ${ }^{a}$, Andrea Sofía Garmendiaa, Carlos Paredes ${ }^{a}$, Miguel Ángel Ropero ${ }^{b, c}$ \\ ${ }^{a}$ Departamento Ingeniería Geológica y Minera, E.T.S.I. de Minas y Energía, Universidad Politécnica de Madrid, C/ Ríos Rosas 21, \\ 28003 Madrid, España. rogelio.delavega@upm.es; andrea2diagon@gmail.com; carlos.paredes@upm.es \\ b Instituto Nacional de Técnica Aeroespacial «Esteban Terradas», Laboratorio de Ingenieros del Ejército «General Marvá», C/ Princesa \\ 36-38, 28008 Madrid, España. roperoama@inta.es \\ c Escuela Politécnica Superior del Ejército, Ministerio de Defensa, C/ Joaquín Costa 6, 28002 Madrid, España. mropaza@et.mde.es
}

\begin{abstract}
:
Deception Island is an active volcano on which two Antarctic bases are located, the Spanish Antarctic Base Gabriel de Castilla and the Deception Antarctic Base, with scientific personnel working there during the summer (December-March). To ensure the safety of these personnel, it is necessary to monitor volcanic and seismic activity continuously with different technologies. Among the technologies that can be used are Differential Synthetic Aperture Radar Interferometry (DInSAR) techniques for ground motion detection. Ground motions can have different origins, including volcanic and seismic movements. In this research we are going to analyse the movements that have occurred throughout 2018, 2019 and 2020, detected using DInSAR techniques and we will try to discriminate their origin. The detection using DInSAR techniques has been carried out with images acquired by the Sentinel 1A and Sentinel 1B satellites during the months of December to March 2018, 2019 and 2020. DInSAR techniques with image pairs have been used. Of all the possible combinations of image pairs, those with the greatest a priori possibilities were selected. Once the image pairs were selected, they were processed using the SNAP (Sentinel Application Platform) and SNAPHU (Statistical-Cost Network-Flow Algorithm for Phase Unwrapping) applications. Once the deformation maps had been obtained, the movements were analysed together with other data on the island such as topographic, geological, geomorphological, slope and orientation maps to find an explanation for each of the movements detected and to classify them according to their type of origin.
\end{abstract}

Key words: Deception Island, DInSAR, landslide, gelifuction

\section{Resumen:}

La isla Decepción es un volcán activo en el cual se localizan dos bases antárticas, la Base Antártica Española Gabriel de Castilla (BAEGC) y la Base Antártica Argentina (BAA), con personal científico que desarrolla su labor durante el verano (diciembre-marzo). Para garantizar la seguridad de dicho personal es necesario monitorizar la actividad volcánica y sísmica de manera continua con distintas tecnologías. Entre las tecnologías que se pueden utilizar se encuentran las técnicas de Interferometría Diferencial SAR (DInSAR) para la detección de movimientos del terreno. Los movimientos en el terreno pueden tener distintos orígenes entre los cuales se encuentran los volcánicos y sísmicos. En esta investigación se van a analizar los movimientos que se han producido a lo largo de los años 2018, 2019 y 2020, detectados mediante técnicas DInSAR y se intentará discriminar su origen. La detección mediante técnicas DInSAR se ha realizado con imágenes adquiridas por los satélites Sentinel 1A y Sentinel 1B durante los meses de diciembre a marzo de los años 2018, 2019 y 2020. Se han utilizado técnicas DInSAR básicas con pares de imágenes. De todas las posibles combinaciones de pares de imágenes se han seleccionado las que tenían mayores posibilidades a priori. Una vez seleccionadas las parejas de imágenes se han procesado utilizando como herramienta las aplicaciones SNAP (Sentinel Application Platform) y SNAPHU (Statistical-Cost Network-Flow Algorithm for Phase Unwrapping). Una vez obtenidos los mapas de deformaciones se han analizado los movimientos junto con otros datos de la isla como los mapas topográficos, geológicos, geomorfológicos, de pendientes y orientaciones con el propósito de encontrar explicación a cada uno de los movimientos detectados y poder clasificarlos por su tipo de origen.

Palabras clave: Isla Decepción, interferometría diferencial SAR, deslizamientos, gelifluxión

\section{Introducción}

En el año 1988 el Ejército de Tierra de España inaugura en la isla Decepción (archipiélago de las islas Shetland del Sur) (Fig. 1) un refugio temporal que denominó
Gabriel de Castilla que en 1998 pasó a denominarse Base Antártica Española Gabriel de Castilla (BAEGC). Partiendo de ese refugio inicial en la BAEGC se han ido añadiendo módulos e instalaciones.

*Corresponding Author: Rogelio de la Vega-Panizo, rogelio.delavega@upm.es 
El seguimiento de la actividad científica de la Base Antártica Española Gabriel de Castilla (BAEGC) en los últimos años ha permitido observar la persistencia de ciertos procesos geológicos activos que se constatan sobre el diario de campaña y que pueden afectar a la estabilidad de las instalaciones. Para poder medir estos lentos procesos activos se necesitan varios años y por lo tanto se propuso un proyecto plurianual (mínimo tres campañas antárticas). Se aprobó el proyecto y estos procesos se están estudiado en la actualidad mediante el proyecto de investigación "Auscultación de procesos activos sobre la ladera y la costa acantilada del entorno próximo a la Base Antártica Española Gabriel de Castilla, Isla Decepción, Antártida".

Este proyecto está liderado y financiado por el Centro LABINGE (Laboratorio de Ingenieros del Ejército "General Marvá")-perteneciente al INTA (Instituto Nacional de Técnicas Aeroespaciales) (LABINGE) junto con el Ejército de Tierra (ET). En él colaboran desde el inicio la Universidad Politécnica de Madrid (UPM-Dpto. de Ing. Geológica y Minera) y la Universidad de Alcalá de Henares (UAH-Dpto. de Geología, Geografía y Medio Ambiente) y a partir del año 2019 se incorpora el Centro Espacial INTA-Torrejón (CEIT) aportando datos SAR del satélite PAZ a través del anunció de oportunidad AO1$P A Z$ en el que participan el equipo de la UPM y del LABINGE.

Entre las tareas básicas del proyecto se encuentra la recogida de datos geotécnicos de campo, en el entorno de la BAEGC. Esta labor la realiza básicamente el personal del LABINGE y ET que se han desplazado a la BAEGC durante las campañas antárticas 2017-2018, 2018-2019 y 2019-2020. Por desgracia, la irrupción de la pandemia COVID-19 en los inicios del 2020 ha supuesto una interrupción en la adquisición de datos al final de la campaña 2019-2020 y en la última campaña 2020-2021.

El objetivo final del proyecto es analizar si existen movimientos de deslizamiento de ladera que afecten a las instalaciones de la BAEGC y si están acoplados con la erosión costera. Para estudiarlos geotécnicamente se propusieron unos objetivos iniciales: determinar la existencia de movimientos superficiales, estimar la profundidad del permafrost y espesor de la capa activa, elaborar un modelo 3D de la ladera y el acantilado y por último desarrollar un modelo de recesión costera.

El área que se analizó en detalle es la ladera situada al sur de la BAEGC (Fig. 2). Dentro del objetivo de determinar la existencia de movimientos superficiales, en la primera campaña (2017-2018) se colocaron unas balizas en la ladera y se midió su posición. En la siguiente campaña $2018-2019$ se volvió a medir su posición. De dichas medidas se concluyó que podía haber movimientos superficiales. En la primera campaña, además de las mediciones topográficas se levantaron ocho perfiles por tomografía eléctrica con el fin de cartografiar en el subsuelo la localización del permafrost (potencia y profundidad) y la potencia de la capa activa.

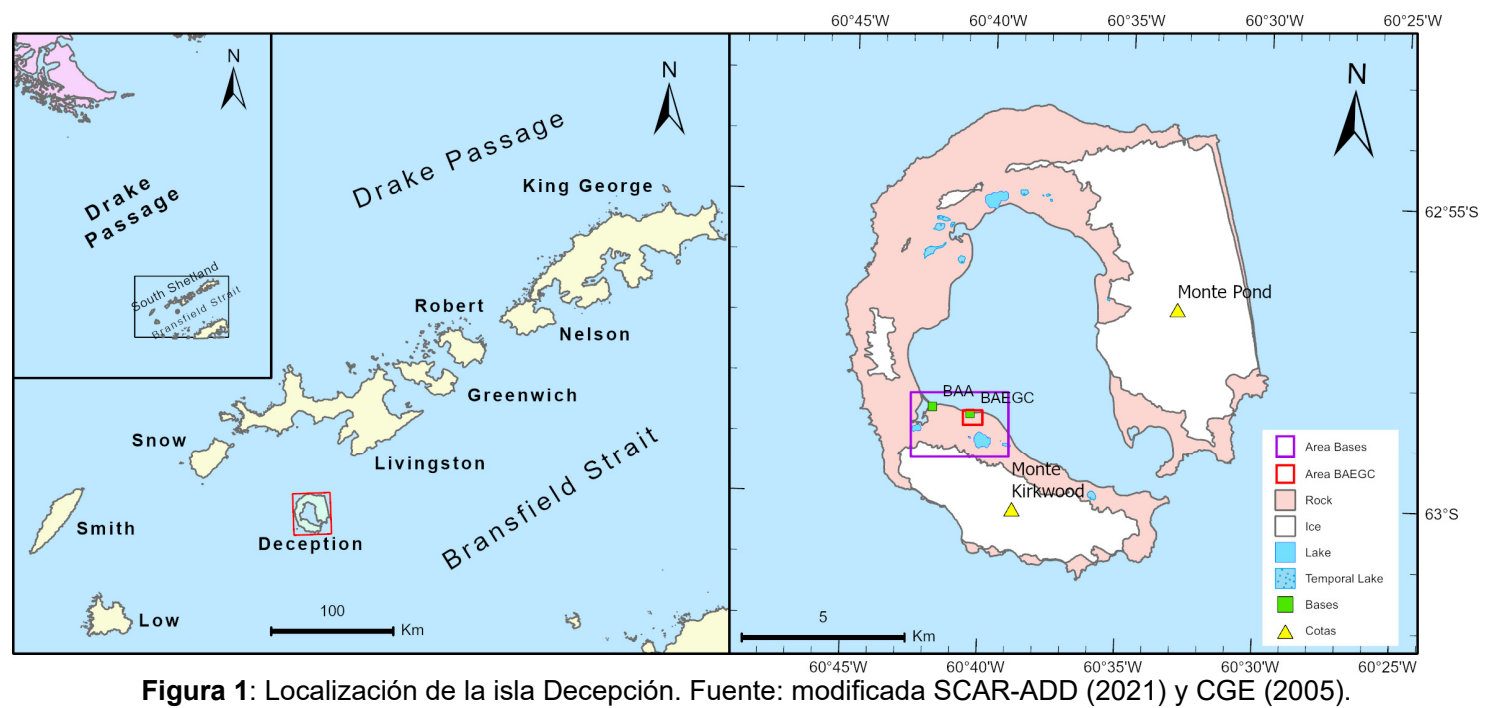

Figura 1: Localización de la isla Decepción. Fuente: modificada SCAR-ADD (2021) y CGE (2005).

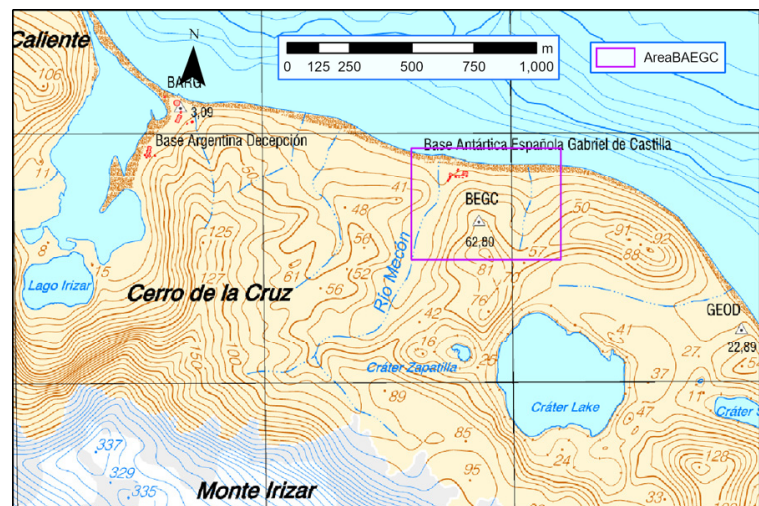

Figura 2: Mapa topográfico del área de las bases BAD y BAEGC. Fuente: modificada CGE (2005).
Una vez constatados que existen movimientos en los terrenos que ocupa la BAEGC, en el año 2019, se decidió estudiar la posibilidad de incluir las técnicas de Interferometría Diferencial SAR (DInSAR) como herramienta de detección temprana de estos movimientos. Para lo cual se decidió participar en el AO1PAZ ofertado por el CEIT. En el marco de esta tarea de estudiar la viabilidad de técnicas DInSAR en la detección de los movimientos en el terreno en primer lugar se utilizaron datos Sentinel 1. Los primeros resultados obtenidos se presentan en este artículo.

Para realizar el análisis DInSAR se ha elegido un área de la isla Decepción que comprende las dos bases científicas que existen actualmente: la Base Antártica Decepción (BAD) gestionada por Argentina y la Base 
Antártica Española Gabriel de Castilla (BAEGC). En la Figura 2 se puede ver un mapa topográfico del área descrita anteriormente. En el mapa se dibuja el área BAEGC que delimita la zona donde se realizó la adquisición de datos geotécnicos del terreno.

\section{Materiales}

Tal y como se expuso en la introducción lo que se busca en el proyecto es analizar los movimientos del terreno en el entorno de la BAEGC a lo largo de varios años con distintas técnicas entre ellas las técnicas DInSAR. Para detectar los movimientos del terreno mediante técnicas DInSAR se han utilizado imágenes SAR obtenidas por los satélites Sentinel 1. En primer lugar, se definió la ventana temporal en la que se iban a adquirir las imágenes. Para poder detectar las deformaciones se necesita que el suelo esté descubierto de nieve y por lo tanto se tienen que utilizar, las imágenes adquiridas durante el verano antártico preferiblemente en las campañas que se habían tomado datos geotécnicos (2017-2018, 2018-2019 y 2019-2020). De las tres campañas se decidió recopilar las imágenes adquiridas por los Sentinel 1 entre el 1 de noviembre y el 30 de abril de los años 2017-2018 20182019 y 2019-2020. En la Tabla 1 se puede ver un listado completo de las imágenes adquiridas en las tres campañas.

Tabla 1: Listado de fechas de adquisición del Sentinel-1.

\begin{tabular}{c|c|c}
$\begin{array}{c}\text { Campaña 2017- } \\
2018\end{array}$ & $\begin{array}{c}\text { Campaña 2018- } \\
2019\end{array}$ & $\begin{array}{c}\text { Campaña 2019- } \\
2020\end{array}$ \\
\hline $07 / 11 / 2017$ & $02 / 11 / 2018$ & $09 / 11 / 2019$ \\
$19 / 11 / 2017$ & $14 / 11 / 2018$ & $21 / 11 / 2019$ \\
$01 / 12 / 2017$ & $26 / 11 / 2018$ & $03 / 12 / 2019$ \\
$13 / 12 / 2017$ & $08 / 12 / 2018$ & $15 / 12 / 2019$ \\
$25 / 12 / 2017$ & $20 / 12 / 2018$ & $27 / 12 / 2019$ \\
$06 / 01 / 2018$ & $01 / 01 / 2019$ & $08 / 01 / 2020$ \\
$18 / 01 / 2018$ & $13 / 01 / 2019$ & $20 / 01 / 2020$ \\
$30 / 01 / 2018$ & $25 / 01 / 2019$ & $01 / 02 / 2020$ \\
$11 / 02 / 2018$ & $06 / 02 / 2019$ & $13 / 02 / 2020$ \\
$23 / 02 / 2018$ & $18 / 02 / 2019$ & $25 / 02 / 2020$ \\
$07 / 03 / 2018$ & $02 / 03 / 2019$ & $08 / 03 / 2020$ \\
$19 / 03 / 2018$ & $14 / 03 / 2019$ & $20 / 03 / 2020$ \\
$31 / 03 / 2018$ & $26 / 03 / 2019$ & $01 / 04 / 2020$ \\
$12 / 04 / 2018$ & $07 / 04 / 2019$ & $13 / 04 / 2020$ \\
$24 / 04 / 2018$ & $19 / 04 / 2019$ & $25 / 04 / 2020$
\end{tabular}

Además de la cobertura nival otros factores que afectan en la detección de los movimientos mediante técnicas DInSAR son la cobertura nubosa y las precipitaciones. Por lo tanto, estos tres factores junto con otros de índole interferométrica se van a utilizar para la selección de las imágenes más adecuadas para cada campaña. En cada una de las tres campañas se va a elegir una pareja de imágenes para realizar el procesado DInSAR.

Tal y como se ha dicho anteriormente, los primeros condicionantes de la elección eran la cobertura nival y la meteorología de la isla en el momento de la adquisición de la imagen. Para estimar estos condicionantes se utilizaron imágenes en el óptico y los datos meteorológicos y de la cobertura de nieve recogidos en los diarios de la BAEGC. Se desecharon todas las imágenes que a priori parecían tener una cobertura nival extensa y/o la meteorología era adversa. El resultado del análisis anterior fue la selección de una serie de imágenes cuyo listado se puede ver en la Tabla 2.

Tabla 2: Listado de imágenes Sentinel-1 descargadas.

\begin{tabular}{c|c|c}
$\begin{array}{c}\text { Campaña 2017- } \\
2018\end{array}$ & $\begin{array}{c}\text { Campaña 2018- } \\
2019\end{array}$ & $\begin{array}{c}\text { Campaña 2019- } \\
2020\end{array}$ \\
\hline $19 / 11 / 2017$ & $02 / 11 / 2018$ & $15 / 12 / 2019$ \\
$25 / 12 / 2017$ & $06 / 02 / 2019$ & $27 / 12 / 2019$ \\
$07 / 03 / 2018$ & $02 / 03 / 2019$ & $08 / 01 / 2020$ \\
$31 / 03 / 2018$ & & $01 / 02 / 2020$ \\
$12 / 04 / 2018$ & & $08 / 03 / 2020$ \\
$24 / 04 / 2018$ & & $13 / 04 / 2020$
\end{tabular}

Con estas imágenes se analizaron los posibles emparejamientos y en función de la meteorología y la línea base espacial y temporal se decidió obtener el interferograma y el mapa de coherencia de los siguientes pares que se listan en la Tabla 3.

Tabla 3: Listado de pares de imágenes Sentinel-1 con interferograma y coherencia.

\begin{tabular}{c|c|c} 
Pares de imágenes & Baseline $(m)$ & Precipitación \\
\hline 19/11/17 12/04/18 & 127.89 & nula/débil \\
25/12/17 12/04/18 & 76.67 & nula \\
$07 / 03 / 18 ~ 31 / 03 / 18$ & 123.09 & alta/nula \\
$07 / 03 / 18 ~ 12 / 04 / 18$ & 13.03 & alta/nula \\
$31 / 03 / 18 ~ 24 / 04 / 18$ & 130.21 & nula \\
$02 / 11 / 18$ 06/02/19 & 165.38 & nula \\
$06 / 02 / 19 ~ 02 / 03 / 19$ & 125.19 & nula/débil \\
15/12/19 01/02/20 & 46.95 & /débil \\
27/12/19 13/04/20 & 203.32 & débil/ \\
$08 / 01 / 20 ~ 13 / 04 / 20$ & 187.94 & Bruma/ \\
$01 / 02 / 20 ~ 08 / 03 / 20$ & 58.59 & débil/nula
\end{tabular}

Se analizaron los interferogramas y los mapas de coherencia de todas estas parejas de imágenes y junto con los datos meteorológicos y las líneas base temporal y espacial se decidió que las tres mejores parejas para analizar en detalle los movimientos eran las siguientes y se pueden ver en las Tablas 4 y 5 .

Estos tres pares se procesaron hasta obtener el mapa con los desplazamientos Line Of Sight (LOS) del terreno.

Los productos imagen SAR del Sentinel 1 utilizados en el procesado interferométrico son datos adquiridos en modo Interferometric Wide Swath (IW), con un nivel L1 de correcciones y en forma de producto Single-Look Complex (SLC) (Bourbigot et al. 2016). Una muestra del tipo de imagen SLC utilizada se puede ver en la Figura 3.

Además de los datos SAR para poder analizar el origen de los desplazamientos detectados mediante las imágenes SAR es necesario conocer la verdad terreno en 
el entorno de las bases. Esta verdad terreno se va a obtener a partir de los datos geotécnicos obtenidos en las campañas, mapas topográficos, el modelo de elevaciones del área, el modelo de pendientes y los mapas geomorfológicos y geológico de la isla.

Tabla 4: Selección final de imágenes.

\begin{tabular}{c|c|c}
$\begin{array}{c}\text { Campaña 2017- } \\
2018\end{array}$ & $\begin{array}{c}\text { Campaña 2018- } \\
2019\end{array}$ & $\begin{array}{c}\text { Campaña 2019- } \\
2020\end{array}$ \\
\hline $31 / 03 / 2018$ & $06 / 02 / 2019$ & $01 / 02 / 2020$ \\
$24 / 04 / 2018$ & $02 / 03 / 2019$ & $08 / 03 / 2020$
\end{tabular}

Tabla 5: Listado de pares de imágenes Sentinel-1.

\begin{tabular}{c|c|c|c}
$\begin{array}{c}\text { Pares de } \\
\text { imágenes }\end{array}$ & $\begin{array}{c}\text { Baseline } \\
(\mathrm{m})\end{array}$ & Precipitación & $\begin{array}{c}\text { Baseline } \\
\text { temporal } \\
\text { (días) }\end{array}$ \\
\hline $31 / 03 / 2018$ & 130.21 & nula & 24 \\
$24 / 04 / 2018$ & 125.19 & nula/débil & 24 \\
$06 / 02 / 2019$ & débil/nula & 36 \\
$02 / 03 / 2019$ & 58.59 & & \\
$01 / 02 / 2020$ & &
\end{tabular}

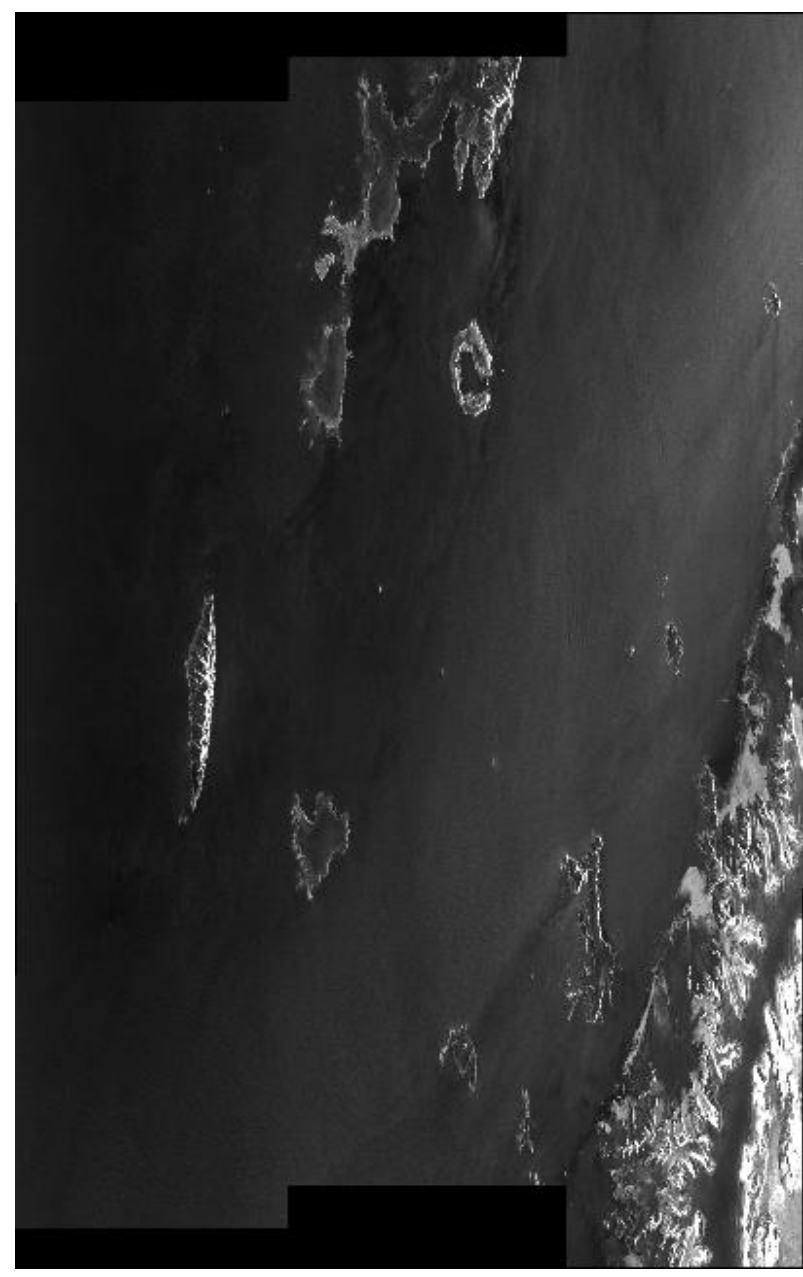

Figura 3: Imagen SLC intensidad 31/03/2018 (ESACopernicus)
De la isla Decepción se ha dispuesto del mapa topográfico escala 1:25000 realizado por el Centro Geográfico del Ejército (CGE), España (CGE 2005). Este mapa se ha utilizado en formato digital ráster y formato digital vectorial. En formato ráster el píxel tiene una resolución de $2.5 \mathrm{~m}$. El formato digital vectorial original era formato CAD Microstation (dgn) que se convirtió en capas vectoriales en formato geodatabase feature de ArcGIS. También se han utilizado los mapas y otros datos geográficos disponibles en los servidores SCAR Antarctic Digital Database (Gerrish et al. 2021).

A partir de las curvas de nivel de este mapa topográfico en formato vectorial un equipo de investigadores de la Universidad de Cádiz (UCA) y del CGE obtuvieron el Modelo Digital de Elevaciones (MDE) de la isla Decepción en formato TIFF. Este MDE tiene un píxel de resolución de $2 \mathrm{~m}$. En la Figura 4 se puede ver el MDE.

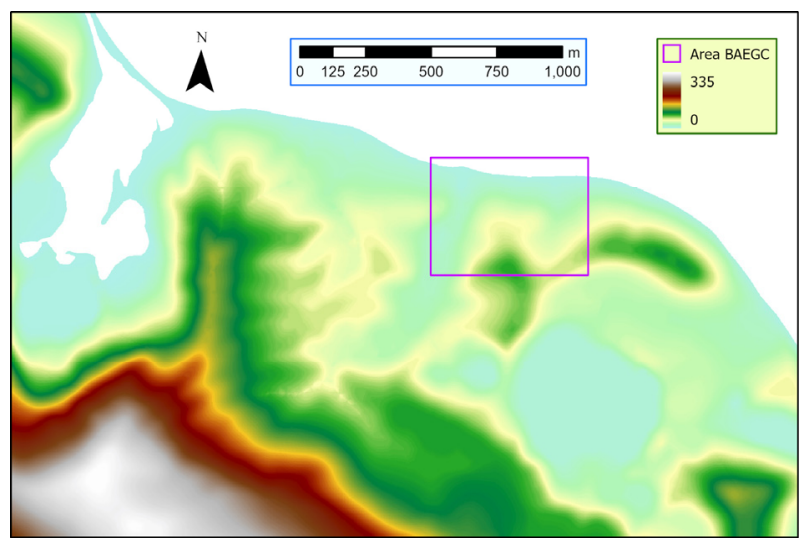

Figura 4: Modelo Digital de Elevaciones.

Este modelo se ha procesado con herramientas de análisis de MDE incluidas en la aplicación ArcGIS para producir el Modelo Digital de Pendientes (MDP) de la isla Decepción. EI MDP se remuestreó para que tuviera un píxel con la misma resolución que los mapas de desplazamientos LOS, $10 \mathrm{~m}$. En la Figura 5 se puede ver el mapa con las pendientes clasificadas según el criterio de la FAO (Baxter 2006).

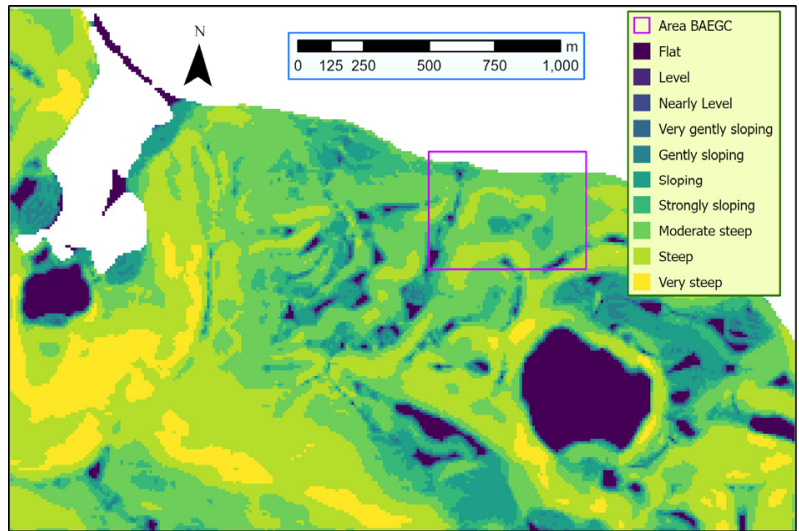

Figura 5: Mapa pendientes.

Respecto a los mapas geológico y geomorfológico se dispone de tres mapas de distintos autores. Los mapas más antiguos son el mapa geológico y el mapa geomorfológico publicados por el British Antarctic Survey (Smellie et al. 2002). Ambos mapas están a escala 1:25000 y se apoyan en la primera edición del mapa 
topográfico realizado por el Centro Geográfico del Ejercito (España) (SGE 1992). El tercer mapa es un mapa geomorfológico de la isla Decepción realizado en una tesis doctoral (Goyanes 2015). Los tres mapas (geológico y los dos geomorfológicos) se han escaneado y georreferenciado obteniéndose tres mapas digitales ráster con un pixel de $2 \mathrm{~m}$. En la Figura 6 se puede ver el mapa geomorfológico de Goyanes (2015).

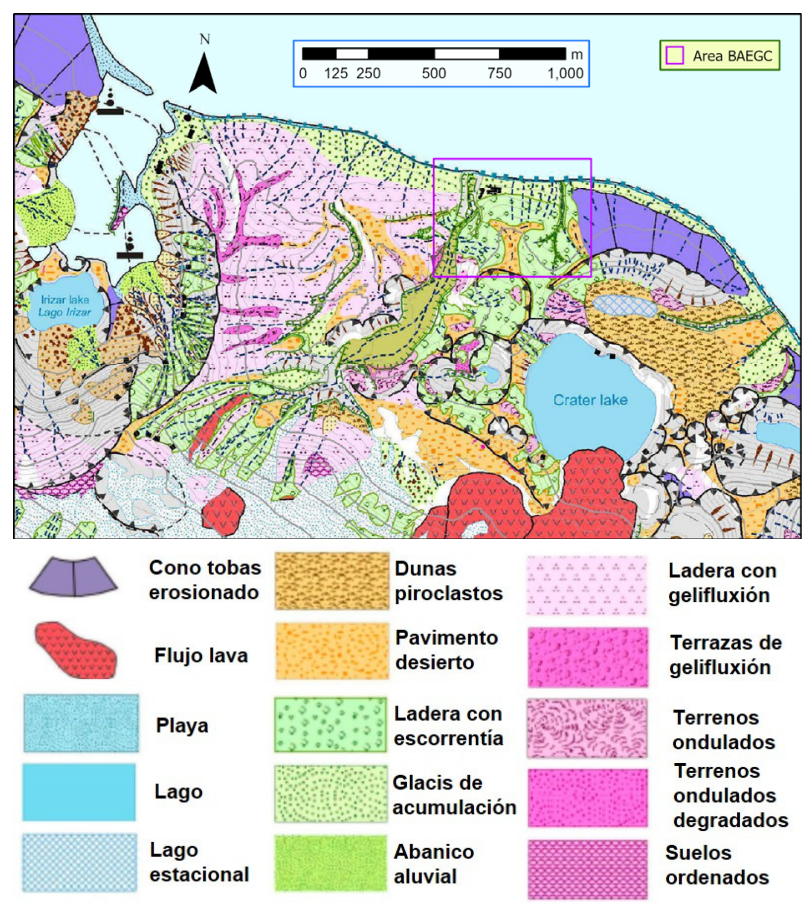

Figura 6: Mapa Geomorfológico (Goyanes 2015).

Otros datos que se han utilizado para analizar los movimientos del terreno han sido los datos topográficos y geofísicos obtenidos en el campo durante las campañas antárticas.

\section{Métodos}

El método de análisis que se ha seguido tiene dos fases. En la primera fase, se han detectado por métodos DInSAR los movimientos en el terreno. Y en una segunda fase, se han comparado dichos movimientos con la información verdad terreno del lugar donde se han detectado obtenida a partir de los mapas topográficos, geológicos y geomorfológicos, imágenes ópticas, datos meteorológicos y datos de campo de la isla Decepción. Se ha intentado dar una explicación a las señales de movimiento detectadas.

La primera fase, procesado DInSAR, se ha realizado con las imágenes radar de la isla Decepción completa. En la última etapa de la fase, de los resultados obtenidos se han extraído los del área de las bases definida al final de la introducción.

El procesado DInSAR de dos imágenes SAR se basa en medir la fase de un mismo punto del terreno en dos épocas distintas desde posiciones espaciales lo más próximas y obtener la diferencia entre las fases medidas. Si el punto no se ha movido la diferencia de fase será nula, pero si el objetivo se ha movido existirá una diferencia de fase $(\Delta \varphi)$. Esta imagen con las diferencias de fase es lo que se denomina interferograma. Esta diferencia en la fase tiene varias componentes una primera es el desplazamiento del objetivo $\left(\varphi_{\text {desp }}\right)$. Una segunda componente contribución de la topografía del terreno y la curvatura de la Tierra $\left(\varphi_{\text {topo }}\right)$. Una tercera componente es la atmosférica $\left(\varphi_{a t m}\right)$. Y la cuarta y última es el ruido $\left(\varphi_{\text {ruido }}\right)$. Esto se puede expresar mediante la siguiente ecuación (Minh et al. 2020), Ec. (1):

$\Delta \varphi=\varphi_{\text {desp }}+\varphi_{\text {topo }}+\varphi_{\text {atm }}+\varphi_{\text {ruido }}$

Dada la ecuación anterior si se desea que el interferograma sirva para obtener los desplazamientos LOS hay que eliminar las otras tres componentes: topográfica, atmosférica y ruido. En el procesado DInSAR que se ha realizado, en primer lugar, se obtuvo el interferograma de los desplazamientos LOS.

Como se comentó anteriormente en la primera fase del análisis se ha trabajado con dos herramientas. En la primera etapa de esta fase se ha utilizado la herramienta proporcionada por la Agencia Espacial Europea (ESA), la aplicación SNAP 8.0 (SentiNel Applications Platform) que incluye la herramienta Sentinel 1 Toolbox (ESA 2021) para obtener el interferograma de cada pareja de imágenes siguiendo los pasos que se pueden ver en la Figura 7.

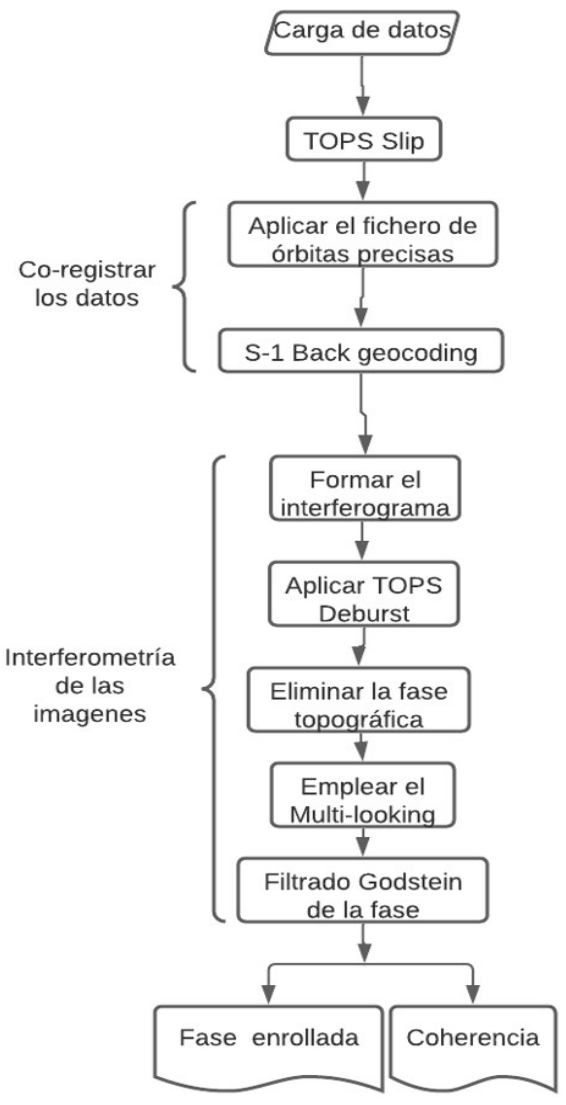

Figura 7: Diagrama de flujo de la primera etapa.

En el primer paso de esta etapa se corregistraron las imágenes, es decir, buscaron los ecos radar procedentes del mismo objetivo. En el siguiente paso se calculó el interferograma. A ese interferograma se le eliminó la componente topográfica utilizando el MDE de la isla. Una vez eliminada la componente topográfica nos queda la atmosférica. 
La componente atmosférica la suponemos muy atenuada debido a la selección de las imágenes con mejor meteorología y por lo tanto se asume como ruido. En los últimos pasos se anula la componente de ruido mediante técnicas de filtrado multilooking y Godstein. El resultado es el interferograma enrollado de los desplazamientos LOS. Los tres interferogramas que se han obtenido se pueden ver en la Figura 8.

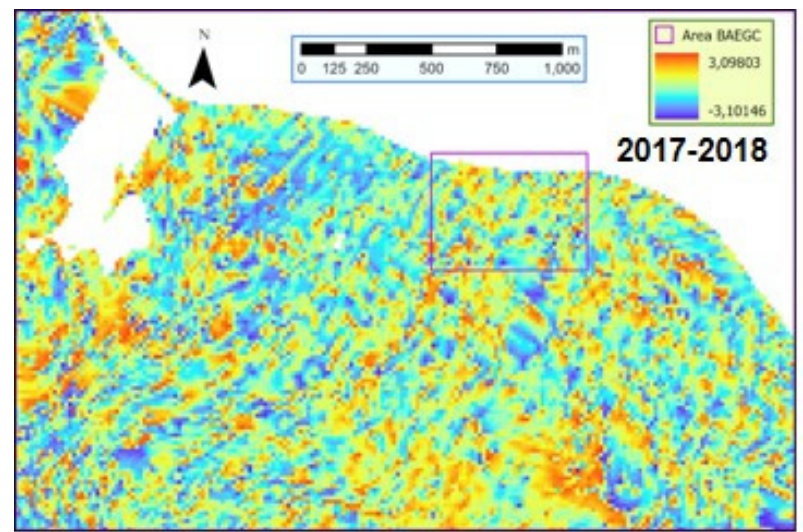

(a)

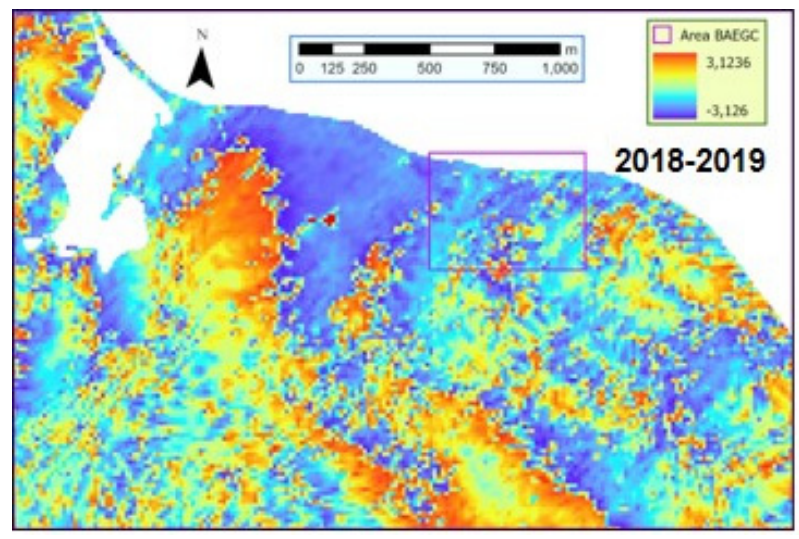

(b)

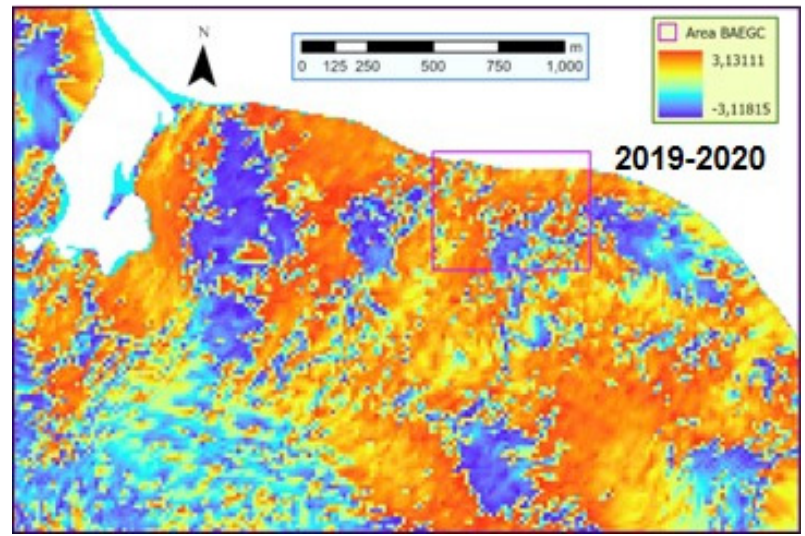

(c)

Figura 8: Interferogramas de los desplazamientos LOS: a) 2017-2018; b) 2018-2019; c) 2019-2020.

A la vez que se obtuvieron los interferogramas se han obtenido los mapas de coherencias que se pueden ver en la Figura 9.

En la segunda etapa, se ha utilizado la aplicación SNAPHU 2.0.4 (Statistical-Cost, Network-Flow Algorithm for Phase Unwrapping) para desenrollar la fase (Chen and Zebker 2000, 2001, 2002).

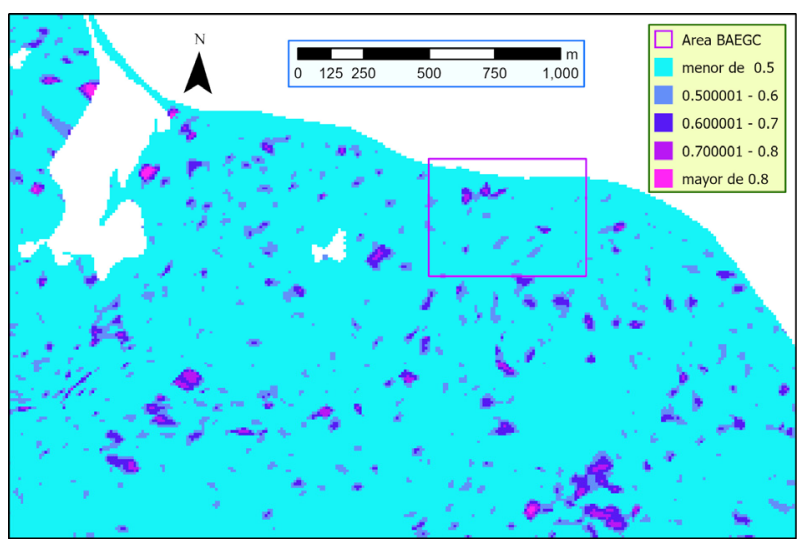

(a)

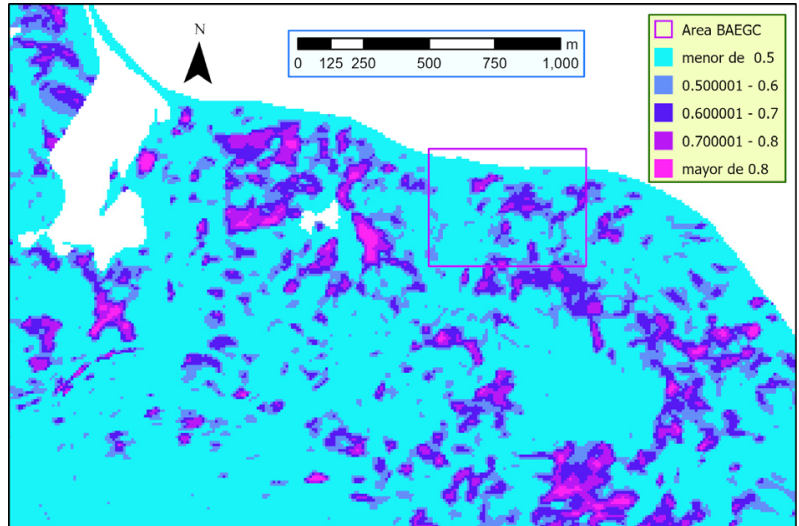

(b)

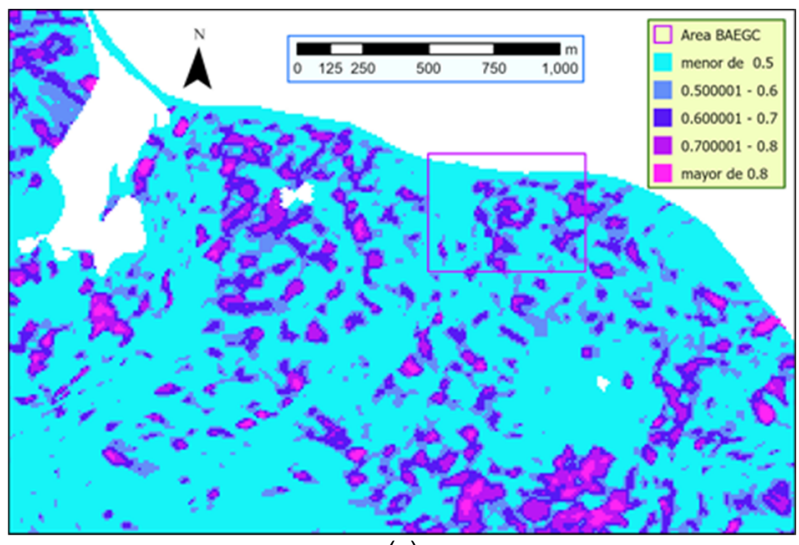

(c)

Figura 9: Mapa de coherencia para las campanas: a) 2018; $2019 ; 2020$.

En la tercera etapa una vez que hemos desenrollado la fase se obtiene el mapa de desplazamientos Line Of Sight (LOS) de la superficie analizada con las herramientas de SNAP 8.0. Los resultados se pueden ver en la Figura 10.

En la cuarta y última etapa, con la aplicación SNAP 8.0, se proyectan cartográficamente (geocodificación) los interferogramas y mapas obtenidos en la etapa anterior para poderlos cotejar con los datos de verdad terreno en la siguiente fase. Estos mapas se exportan en formato TIFF y se recortan al área en el entorno a las bases antárticas. Estas imágenes en el entorno de las bases son las que se muestran en las Figuras 8 - 10.

En la Figura 11 se puede ver un diagrama de flujo con las tres últimas etapas. 


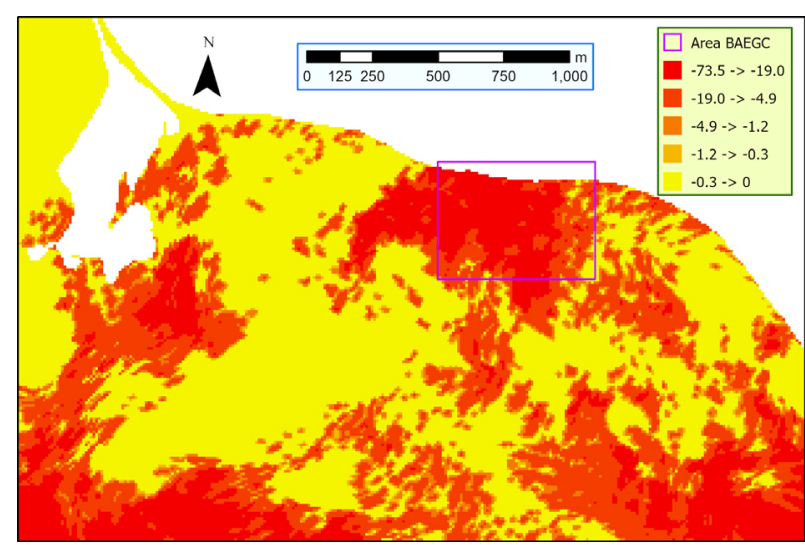

(a)

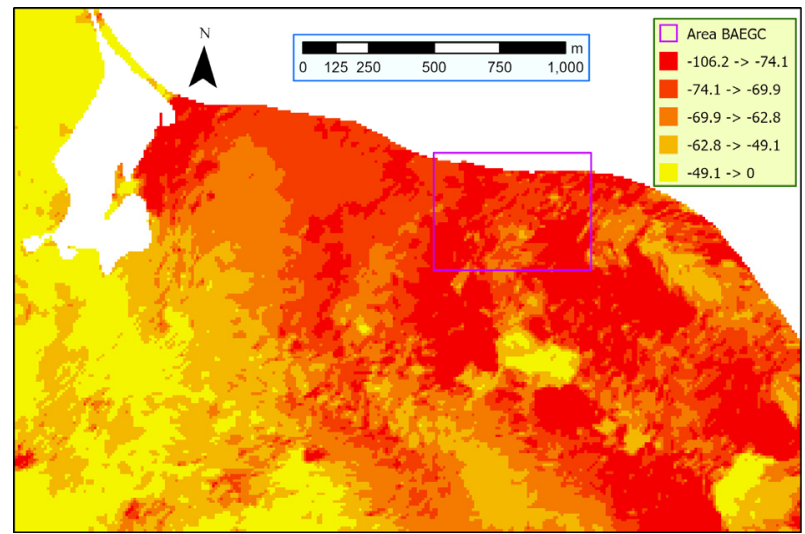

(b)

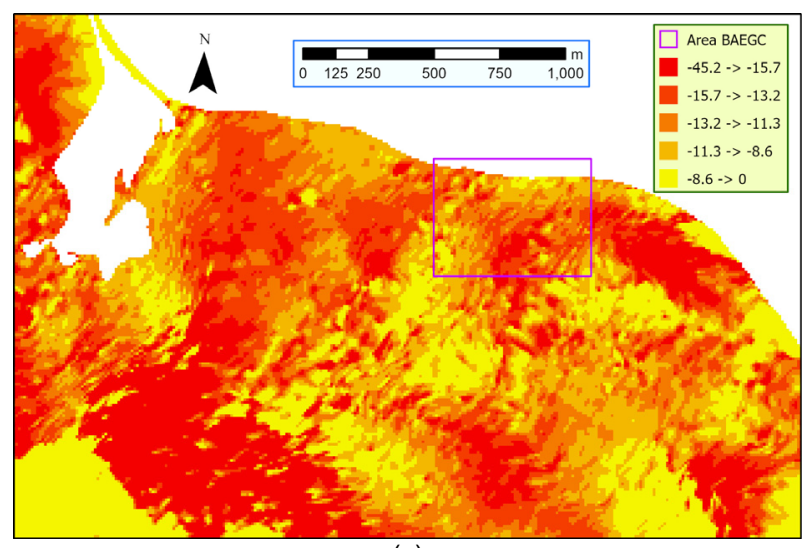

(c)

Figura 10: Mapa desplazamientos LOS en $\mathrm{mm}$ para las campañas: a) 2018; b) 2019; c) 2020.

En resumen, en esta primera fase se ha realizado el procesado DInSAR a las tres parejas de imágenes previamente descritas en el apartado de materiales según el algoritmo descrito anteriormente y se ha obtenido como resultado un mapa de desplazamientos LOS y un mapa de coherencia para cada una de las tres campañas analizadas, en el entorno de las bases antárticas. Para ejecutar esta primera fase se ha seguido varios documentos y manuales (Braun and Veci 2020).

\section{Resultados}

Los resultados obtenidos en esta primera fase son los mapas de coherencia y los mapas de desplazamientos LOS.

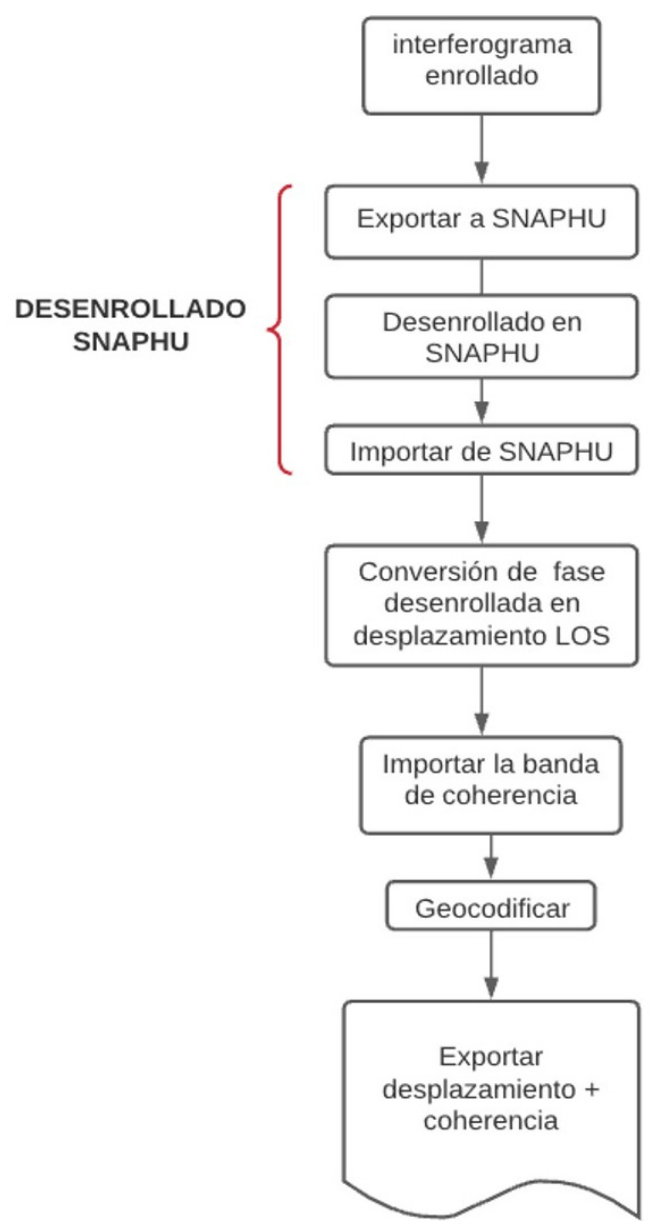

Figura 11: Diagrama de flujo de las etapas 2, 3 y 4.

Los mapas de coherencia son un estimador, en el sentido estadístico, de la calidad de la medida de la fase. Este parámetro varía entre 0 y 1 siendo 0 la falta de coherencia en la fase y 1 coherencia total. Por lo tanto, la estimación de los desplazamientos LOS en un pixel, a priori, tiene una mayor calidad cuanto mayor es la coherencia en dicho pixel.

En la Figura 9 vemos que esta coherencia varia de una campaña a otra. Para representar de una manera más visual la coherencia se ha clasificado los valores en 5 clases siendo la clase color azul claro la de coherencia menor o igual a 0.5 las restantes 4 clases de color azul más oscuro, violeta y magenta los valores mayores de 0.5 hasta 1.0.

El segundo grupo de resultados obtenidos son los mapas de desplazamientos LOS. Para su mejor análisis se han simbolizado mediante una escala amarillo-rojo en la que el amarillo es un desplazamiento nulo y el rojo es el desplazamiento máximo alejándose de la antena. La agrupación de las medidas en clases se ha obtenido dividiendo el rango de los datos en quintiles.

En la primera campaña (2017-2018) con una base temporal de 24 días se obtuvieron unos desplazamientos LOS entre 0 y $-73 \mathrm{~mm}$ alejándose de la antena (signo negativo) que se pueden ver en la Figura 10a.

En la segunda campaña (2018-2019) con una base temporal de 24 días se obtuvieron unos desplazamientos LOS entre 0 y $-106 \mathrm{~mm}$ predominando valores por encima de los $-60 \mathrm{~mm}$ que se pueden ver en la Figura $10 \mathrm{~b}$. 
En la tercera campaña (2019-2020) con una base temporal de 36 días se midieron unos desplazamientos LOS entre 0 y $-45 \mathrm{~mm}$. que se pueden ver en la Figura 10c.

\section{Discusión y conclusiones}

En la segunda fase del trabajo se analizaron los resultados obtenidos en el entorno de las bases antárticas mediante con ayuda de las herramientas de ArcGIS. Para realizar el análisis de una manera más detallada se han enmascarado los valores de desplazamiento LOS con la coherencia de manera que únicamente se tienen en cuenta los pixeles cuya coherencia es mayor de 0.5. En las Figuras 12 - 14 se representan en cyan los píxeles enmascarados y en la escala amarillo-rojo los pixeles cuyo desplazamiento LOS se ha analizado.

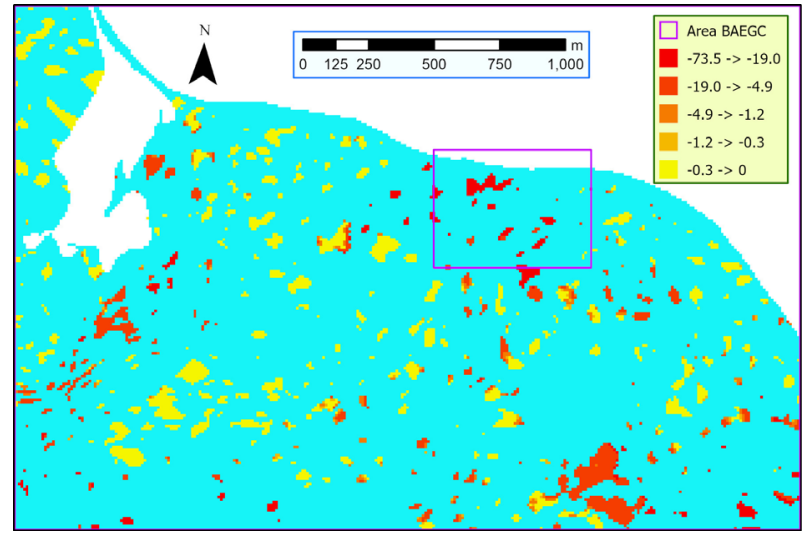

Figura 12: Desplazamientos LOS con coherencia $>0.5$. Campaña 2017-2018.

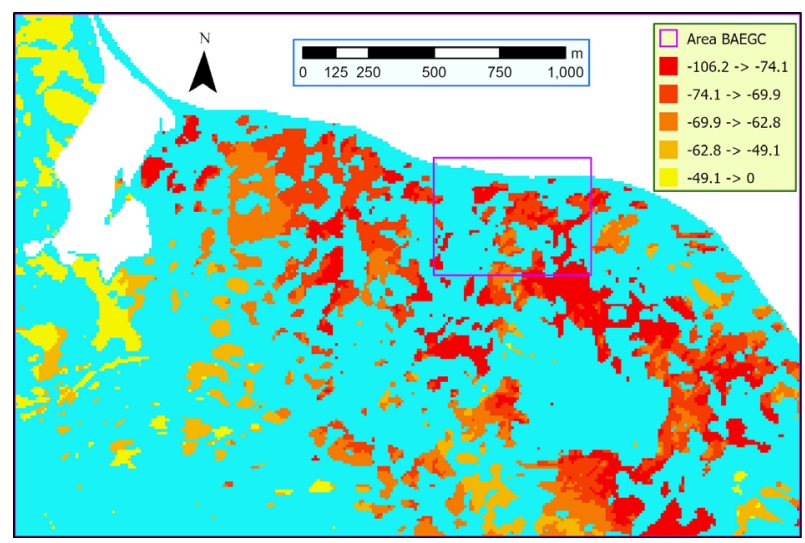

Figura 13: Desplazamientos LOS con coherencia > 0.5 . Campaña 2018-2019.

De estos análisis, llama la atención la gran variabilidad en los desplazamientos LOS del terreno para las distintas campañas. En la primera campaña de 2018 aunque los valores varían entre 0 y $-73 \mathrm{~mm}$ se puede observar que la mayoría de los pixeles con coherencia superior a 0.5 (Fig. 12) tienen valores de desplazamiento en rango menor de $1 \mathrm{~mm}$ (los dos quintiles mas bajos). En cambio, en el entorno de los edificios de la BAEGC en zonas de alta coherencia los valores de desplazamiento LOS están en el quintil más alto $(-19 \mathrm{~mm}$ a $-73 \mathrm{~mm})$.

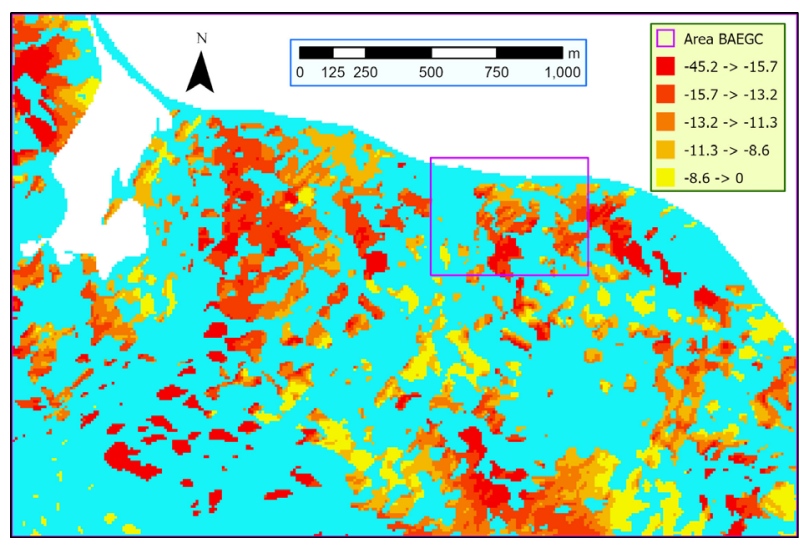

Figura 14: Desplazamientos LOS con coherencia $>0.5$. Campaña 2019-2020.

En la segunda campaña de 2019 los desplazamientos LOS varían entre 0 y $-106 \mathrm{~mm}$. En las zonas de alta coherencia en el entorno de la BAEGC los valores mayoritariamente pertenecen a los dos quintiles superiores $(-74 \mathrm{~mm}$ a $-106 \mathrm{~mm})$. Además, las áreas de alta coherencia son más numerosas que en la campaña de 2018.

En la tercera campaña de 2020 los desplazamientos LOS varían entre 0 y $-45 \mathrm{~mm}$. Se encuentran más distribuidos entre las distintas clases, aunque predominan los valores de los tres quintiles superiores (varían entre $-11 \mathrm{~mm}$ y $45 \mathrm{~mm}$ ). Las áreas de coherencia superior a 0.5 son muy parecidas a las de la segunda campaña.

Es importante destacar que estos desplazamientos de terreno están medidos en la dirección del Line Of Sight (LOS) y por lo tanto dependen de la orientación del terreno respecto de la dirección de iluminación del radar.

Comparando los mapas de desplazamientos con el mapa geomorfológico de Goyanes (2015) se puede observar que gran parte de los desplazamientos se detectan en zonas con procesos de gelifluxión. Comparando con el mapa de pendientes clasificado según Baxter (2006) se detectan la mayoría de los movimientos en laderas con pendientes superiores al $1 \%$ lo cual incluiría movimientos debidos a gelifluxión o a erosión. Comparando las tres campañas se aprecian fuertes diferencias principalmente entre la primera campaña y las otras dos campañas. Todas estas circunstancias parecen indicar que los movimientos detectados son de carácter local, y que pueden ser debidos a variaciones en la capa activa del permafrost y/o procesos de erosión.

En conclusión, con estas tres estimaciones lo único que se puede afirmar es que en el entorno de la BAEGC hay movimientos del terreno y que estos son variables en cada campaña. Por lo tanto, se ha confirmado la existencia de movimientos en el terreno con otra técnica distinta. Para poder cuantificarlos de una manera precisa sería necesario procesar series de imágenes mediante técnicas avanzadas DINSAR. 


\section{References}

BAXTER, S., 2006. Guidelines for Soil Description. Rome: Food and Agriculture Organization of the United Nations. Experimental Agriculture, 43(2), pp. 263-264.

BOURBIGOT, M., JOHNSEN, H. and PIANTANIDA, R., 2016. Sentinel-1 Product Definition (esa.int). Available: https://sentinel.esa.int/documents/247904/1877131/Sentinel-1-Product-Definition [7/15, 2021].

BRAUN, A. and VECI, L., 2020. TOPS Interferometry Tutorial, Sentinel-1 Toolbox Skywatch ESA. Available: http://step.esa.int/docs/tutorials/S1TBX TOPSAR Interferometry with Sentinel-1 Tutorial_v2.pdf [7/15, 2021].

CGE. 2005. Mapa Topográfico de la Isla Decepción - edicion 2a.

CHEN, C. W., and ZEBKER, H. A., 2000. Network approaches to two-dimensional phase unwrapping: intractability and two new algorithms. JOSA A, 17(3), pp. 401-414. DOI: 10.1364/JOSAA.17.000401

CHEN, C. W., and ZEBKER, H. A., 2001. Two-dimensional phase unwrapping with use of statistical models for cost functions in nonlinear optimization. JOSA A, 18(2), 338-351. DOI: 10.1364/JOSAA.18.000338

CHEN, C. W., and ZEBKER, H. A., 2002. Phase unwrapping for large SAR interferograms: Statistical segmentation and generalized network models. IEEE Transactions on Geoscience and Remote Sensing, 40(8), pp. 1709-1719. DOI: 10.1109/TGRS.2002.802453

ESA. 2021. European Space Agency. Sentinel-1 Toolbox - STEP (esa.int). Available: https://step.esa.int/main/toolboxes/sentinel-1-toolbox/ [7/15, 2021].

GERRISH, L., FRETWELL, P. and COOPER, P., 2021. High resolution vector polygons of the Antarctic coastline (7.4), UK Polar Data Centre, Natural Environment Research Council, UK Research \& Innovation. DOI: 10.5285/cdeb448d10de-4e6e-b56b-6a16f7c59095.

GOYANES, G. A., 2015. Control climático e interacciones permafrost-volcanismo, Isla Decepción, Antártida (Doctoral dissertation, Universidad de Buenos Aires. Facultad de Ciencias Exactas y Naturales).

MINH, D. H. T., HANSSEN, R. AND ROCCA, F., 2020. Radar interferometry: 20 years of development in time series techniques and future perspectives. Remote Sensing, 12(9), pp. 1-18. DOI: 10.3390/RS12091364.

SGE. 1992. Mapa Topográfico de la Isla Decepción - edicion 1a.

SMELLIE, J. L., LÓPEZ-MARTÍNEZ, J., HEADLAND, R. K., HERNÁNDEZ-CIFUENTES, F., MAESTRO, A., MILLAR, I. L.; REY, J.; SERRANO, E.; SOMOZA, L.; and THOMSON, J. W., 2002. Geology and geomorphology of Deception Island. Cambridge, British Antarctic Survey, 77pp. (BAS Geomap Series, Sheets 6A and 6B). 